\title{
Implications of COPD in patients admitted to the intensive care unit by community-
} acquired pneumonia

\author{
J. Rello*, A. Rodriguez*, A. Torres”, J. Roig", J. Sole-Violan+, J. Garnacho-Montero", \\ M.V. de la Torre ${ }^{f}$, J.M. Sirvent** and M. Bodi* for the CAPUCI study investigators
}

ABSTRACT: The mortality rate of chronic obstructive pulmonary disease (COPD) patients with community-acquired pneumonia (CAP) is reported to be low. However, studies carried out to date have included $<\mathbf{2 0} \%$ of critically ill patients.

The current authors performed a secondary analysis of a prospective study evaluating 428 immunocompetent patients admitted to the intensive care unit (ICU) for severe CAP.

In total, 176 COPD patients were compared with 252 non-COPD patients. In COPD patients, ICU mortality (odds ratio (OR) 1.58; 95\% confidence interval (CI) 1.01-1.43) and mechanical ventilation (OR 2.78; 95\% Cl 1.63-4.74) rates were higher than in non-COPD patients. The ICU mortality was $39 \%$ for COPD patients initially intubated and $50 \%$ for those who failed noninvasive ventilation. The proportion of patients who were males, aged $\geqslant 70 \mathrm{yrs}$, smokers and who had chronic heart disease or Pseudomonas aeruginosa were higher in COPD patients. Inappropriate empirical antibiotic therapy was associated with higher mortality (OR 3.8; 95\% Cl 1.19-12.6). ICU mortality in COPD patients with adequate therapy was associated with bilateral pneumonia (OR 2.32; 95\% Cl 1.18-4.53) and shock (OR 3.53; 95\% Cl 1.31-9.71).

In conclusion, chronic obstructive pulmonary disease patients hospitalised with communityacquired pneumonia in the intensive care unit had higher mortality and need of mechanical ventilation when compared with patients without chronic obstructive pulmonary disease.

\section{KEYWORDS: Community-acquired pneumonia}

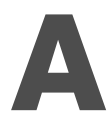
strong association between chronic obstructive pulmonary disease (COPD) and severe community-acquired pneumonia (SCAP), mainly caused by Streptococcus pneumoniae or Haemophilus influenzae, has been suggested [1, 2]. For COPD in particular, this elevated incidence appears to be explained by altered pulmonary defence mechanisms [3]. FEKETY et al. [4] reported a higher incidence of pneumococcal pneumonia in COPD patients than in control patients.

COPD is one of the most frequent comorbidities in patients admitted to hospital for CAP and respiratory failure [1, 5-11]. In a prospective study of SCAP in 529 patients in 33 intensive care units (ICUs) in Spain, COPD was the most frequent comorbidity [12]. Therefore, a secondary analysis was conducted with the primary objective of comparing the demographic characteristics, aetiology and outcome of patients admitted to the ICU for SCAP with and without a prior diagnosis of COPD. A specific goal was to examine if the presence of COPD was a predictor for increased mortality in ICU patients hospitalised for pneumonia. A secondary objective was to assess how the use of noninvasive ventilation (NIV) influenced outcome. The current authors' hypothesis was that COPD patients with SCAP will have a higher ICU mortality and risk of mechanical ventilation than other immunocompetent patients hospitalised in the ICU with SCAP without COPD.

\section{METHODS}

Full details of the methodology used in this prospective, multicentre, observational study have been presented elsewhere [12]. Between December 1, 2000 and February 28, 2002, 529 consecutive patients with CAP admitted to ICUs in 33 hospitals in Spain were enrolled. Institutional review board approval was obtained in accordance with local requirements. Patients were observed until death or ICU discharges.

The enrolled patients were consecutive cases aged $\geqslant 18 \mathrm{yrs}$, with conclusive evidence of pneumonia as primary diagnosis, confirmed by

\section{AFFILIATIONS}

*Hospital Joan XXIII, Universitat Rovira i Virgili/Institut Pere Virgili, Tarragona,

"Hospital Clinic, Barcelona, +Hospital Dr. Negrín, Las Palmas de Gran Canária,

${ }^{\S}$ Hospital Virgen del Rocio, Sevilla, ${ }^{f}$ Hospital Virgen Victoria, Málaga, and

**Hospital Joseph Trueta, Girona,

Spain.

"Hospital Verge de Meritxell, Andorra.

CORRESPONDENCE

J. Rello

Critical Care Dept Joan XXIII University Hospital Carrer Mallafre Guasch 4 E43007 Tarragona

Spain

Fax: 34977295878

E-mail: jordi.rello@urv.net

Received:

November 252005

Accepted after revision:

February 072006

SUPPORT STATEMENT

This study was supported in part by 2005/SGR/920, RED RESPIRA (ISCiii-RTIC 03/11), Marató TV3 and FISS (PI04/1500). The CAPUCI study investigators are listed in the Acknowledgements section.

European Respiratory Journal Print ISSN 0903-1936 Online ISSN 1399-3003 
chest radiography. The study focused on patients in the ICU and excluded patients with respiratory infections other than pneumonia (for example, exacerbated COPD patients), and those on home oxygen therapy. Immunocompromised patients (i.e. patients with HIV infection, neoplasia, those taking cytotoxic drugs or long-term oral steroid therapy, such as daily doses of $>20 \mathrm{mg}$ of prednisolone or the equivalent for $>2$ weeks) were excluded, as well as patients with asthma, interstitial lung diseases or aspiration pneumonia. Patients with COPD criteria were enrolled in this sub-study. Appropriate therapy was defined as the use of at least one antibiotic to which all isolates were susceptible in vitro or (for Pneumocystis jiroveci or Legionella pneumophila) were expected to be susceptible. Pre-existing COPD was defined as a disease state characterised by the presence of airflow limitation due to chronic bronchitis or emphysema. The airflow obstruction may be accompanied by airway hyperreactivity and may be partially reversible [13]. Details of other definitions, including pneumonia or shock, among others, have been reported elsewhere [12]. A patient was considered to be a smoker if they had smoked more than one packet per day within the past 10 yrs [14]. Mechanical ventilation included invasive ventilation and NIV.

A wide range of demographic, clinical and laboratory measures were recorded in each patient, as described previously. In the current study, particular emphasis was placed on the age of the patients, history of smoking, presence of other comorbidities, and severity of illness measured by the acute physiological score (APS) of Acute Physiology And Chronic Health Evaluation (APACHE) II score.

Patients in whom standard treatment or NIV was not successful underwent endotracheal intubation. Variables considered as indicating unsuccessful standard treatment or NIV have been reported previously [15] and included: failure to maintain an arterial oxygen tension/inspiratory oxygen fraction of $>85$; need to protect the airways; inability to spontaneously clear tracheal secretions; agitation requiring sedation; inability to tolerate the face mask; an increase in the arterial carbon dioxide accompanied by a $\mathrm{pH}<7.20$; or septic shock. Treatment decisions for all study participants, including determination of the need for intubation or mechanical ventilation, were made by the primary clinical teams.

An organism was considered to be a "definitive" aetiological agent only if it was isolated from blood or pleural fluid, or if serological tests revealed a four-fold increase in antibody levels. Isolation of P. jiroveci or culture of L. pneumophila or Mycobacterium tuberculosis was considered the basis of a "definitive" diagnosis, in accordance with current guidelines $[16,17]$. Microorganisms isolated from other cultures, including bronchoscopic samples, or with a positive urinary antigen test were considered as "probable" [16, 17].

Discrete variables were expressed as counts (\%) and continuous variables as mean $\pm \mathrm{SD}$, unless stated otherwise. All statistical tests were two-sided. Differences in categorical variables were calculated using a two-sided likelihood ratio Chi-squared test for nondichotomous categorical variables and a two-sided Fisher's exact test for dichotomous variables. The Kaplan-Meier estimate-of-survival curve was used to determine the probability of survival for COPD and nonCOPD patients at 28 days. Survival curves were compared using the log-rank test. Cox proportional hazards regression was used to adjust for severity of the impact of independent variables on ICU mortality. Factors associated with increased mortality by bivariate analysis $(p \leqslant 0.05)$ were entered into the model. A p-value $<0.05$ was considered as significant. In addition to p-values, odds ratios (OR) and 95\% confidence intervals (CI) were calculated.

\section{RESULTS}

In total, 529 patients were recruited for the original study [8]. Of these, $10(1.9 \%)$ were excluded due to aspiration pneumonia, $22(4.1 \%)$ due to HIV and $69(13.0 \%)$ due to other causes of immunocompromise. The remaining 428 immunocompetent patients were available for analysis. The 176 (41.1\%) COPD patients, 107 of whom were smokers, were the subject of the current study. Differences in baseline characteristics between COPD and non-COPD patients are summarised in table 1 . The ICU mortality rate was significantly higher $(30.1 \%$ versus $21.4 \% ; \mathrm{p}=0.05)$ in COPD than in non-COPD patients. A Kaplan-Meier survival curve (fig. 1) shows a significant difference (Log rank 3,69; $\mathrm{p}=0.05$ ) in 28-day mortality for patients with and without COPD.

Fourteen $(15.7 \%)$ out of the 89 COPD patients with microbiological diagnosis received inadequate empirical therapy and eight (57.1\%) died, compared with 19 deaths $(25.7 \%$; $\mathrm{p}<0.05)$ in COPD patients with adequate empirical regimen. Microorganisms associated with inadequate empirical therapy are detailed in table 2 . The baseline characteristics of the remaining 162 COPD patients (with adequate empirical therapy and ventilated $>24 \mathrm{~h}$ ) according to the outcome are shown in table 3. ICU mortality in COPD patients was associated with higher APS at day $1(\mathrm{p}=0.01)$, bilateral pneumonia $(p<0.01)$, bacteraemia $(p=0.04)$, shock $(p=0.01)$, mechanical ventilation $(p=0.001)$ and neurological illness $(p=0.01)$. In this group, the Cox proportional regression analysis demonstrated that ICU mortality was independently associated with shock (OR 3.5) and bilateral pneumonia (OR 2.3). The same variables plus APS score (OR 1.06) and age (OR 1.03) were also identified for non-COPD episodes (table 4).

ICU mortality was not different for ventilated COPD and nonCOPD patients $(34.2 \%$ versus $28.9 \%$; $p=0.36)$. Table 5 compares characteristics and outcomes of patients according to ventilation subgroups. In COPD patients, 105 underwent endotracheal intubation within the first hours of ICU admission, 41 $(39 \%)$ of whom died. There were $50(28.4 \%)$ patients assigned to NIV. Of these patients, $24(13.6 \%)$ required further intubation, and $12(50 \%)$ of these died. The mortality rate in COPD patients with failure to NIV compared with COPD patients who underwent initial intubation had a relative risk of 1.56 (95\% CI 0.6-3.8). The Kaplan-Meier survival plot at 28 days for initial intubation versus delayed intubation due to unsuccessful NIV is shown in figure $2(\log \operatorname{rank} 1.58 ; \mathrm{p}=0.23)$. All COPD patients with NIV who did not require intubation were discharged alive. COPD patients with successful NIV had a mean \pm SD ICU length of stay of $8.1 \pm 7.1$ days compared with $17.8 \pm 14.0$ days $(\mathrm{p}<0.05)$ for COPD intubated patients who survived. 


\begin{tabular}{|c|c|c|c|c|}
\hline Variable & COPD patients & Non-COPD patients & $p$-value & OR $(95 \% \mathrm{Cl})$ \\
\hline Age yrs & $67.1 \pm 10.1$ & $57.1 \pm 17.6$ & $<0.001$ & \\
\hline$>70$-yrs old & $84(47.7)$ & $75(29.8)$ & $<0.001$ & \\
\hline \multicolumn{5}{|l|}{ Comorbidities } \\
\hline Smoker & $107(60.8)$ & 97 (38.5) & $<0.001$ & $2.48(1.67-3.68)$ \\
\hline Chronic heart disease & 75 (42.6) & $59(23.4)$ & $<0.001$ & $2.43(1.60-3.69)$ \\
\hline Alcoholism & $52(29.5)$ & $59(23.4)$ & 0.18 & $1.37(0.88-2.12)$ \\
\hline Diabetes mellitus & $44(25.0)$ & $58(23.0)$ & 0.72 & $1.11(0.71-1.75)$ \\
\hline Stroke & $13(7.4)$ & $22(8.7)$ & 0.74 & $0.83(0.40-1.70)$ \\
\hline Mechanical ventilation & $155(88.0)$ & $183(72.6)$ & $<0.001$ & $2.78(1.62-4.74)$ \\
\hline VAP & $10(5.6)$ & $21(8.3)$ & 0.39 & $0.66(0.30-1.44)$ \\
\hline ICU mortality rate & $53(30.1)$ & $54(21.4)$ & 0.05 & $1.58(1.01-1.43)$ \\
\hline LOS for survivors & $13.7 \pm 12.6$ & $16.8 \pm 17.9$ & 0.04 & \\
\hline
\end{tabular}

Data are presented as $n$, mean \pm SD or $n(\%)$, unless otherwise stated. OR: odds ratio; Cl: confidence interval; APS: acute physiological score of Acute Physiology And Chronic Health Evaluation II score; VAP: ventilator-associated pneumonia; ICU: intensive care unit; LOS: length of stay. "\#: excluded patients with immunocompromise.

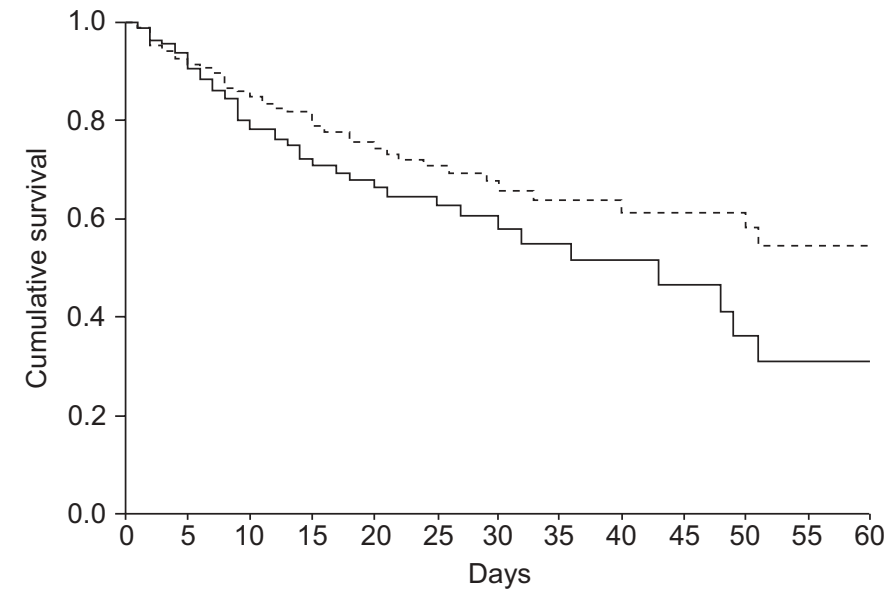

FIGURE 1. Kaplan-Meier survival curve for the proportion of surviving hospitalised community-acquired pneumonia patients with (-) and without (- - -) the presence of chronic obstructive pulmonary disease.

Microbiological documentation (definite and probable) was achieved in $89(50.5 \%)$ COPD patients and is detailed in table 6. S. pneumoniae was the most common pathogen both in patients with and without COPD. Pseudomonas aeruginosa was isolated with significantly higher frequency in COPD patients than non-COPD patients $(14.6 \%$ versus $0.8 \%$; $<<0.05)$. No clinical variable was associated with $P$. aeruginosa in the subset of COPD patients. Overall, S. pneumoniae represented $54.1 \%$ of isolates in COPD patients, followed by $P$. aeruginosa (13.5\%), Haemophilus influenzae $(11.4 \%)$ and L. pneumophila $(4.1 \%)$.
Invasive diagnostic methods were performed in $68(38.6 \%)$ COPD patients, whereas 20 microorganisms (17 S. pneumoniae and three L. pneumophila) were diagnosed on the basis of urinary antigen tests. Four COPD patients had pneumococcal empyema. The microorganisms isolated in COPD patients who died were as follows: $S$. pneumoniae $(\mathrm{n}=16)$; $P$. aeruginosa $(\mathrm{n}=7)$; M. tuberculosis $(\mathrm{n}=2)$; S. aureus $(\mathrm{n}=1)$; K. pneumoniae $(\mathrm{n}=1)$; and Proteus spp. $(\mathrm{n}=1)$.

The most frequent antibiotic treatments in COPD patients were: cephalosporines plus macrolide $(n=93,52.9 \%)$, cephalosporines; plus fluoroquinolone $(\mathrm{n}=33,18.8 \%)$, other combinations $(n=9,5.2 \%)$; and monotherapy $(n=41,23.8 \%)$. Time until the first antibiotic dose was estimated to be $6.6 \pm 10.1 \mathrm{~h}$. Details of antibiotic regimens are shown in table 2. In total, 80 $(45.4 \%)$ COPD patients received empirical anti-pseudomonal therapy and the ICU mortality rate $(32.5 \%)$ was similar to episodes receiving other regimens (28.1\%). No differences in ICU mortality rate were observed between COPD patients who received combination therapy or monotherapy $(30.4 \%$ versus $29.3 \% ; \mathrm{p}=0.95)$. ICU mortality rate did not significantly differ with the use of a fluoroquinolone ( $31.4 \%$ versus $29.5 \%$; $p=0.93$ ) or a macrolide $(26.5 \%$ versus $34.6 \%$; $p=0.31)$ as part of the empirical regimen. Finally, antibiotic treatment was modified in $77(43.7 \%)$ episodes: 23 due to de-escalation, 14 due to inappropriate treatment and 40 due to poor resolution (table 2). A bacterial pathogen was identified in 17 (12 caused by $S$. pneumoniae) of these episodes with changes of antibiotic therapy due to poor resolution and $20(50 \%)$ of them ultimately died.

\section{DISCUSSION}

The findings from this large, multicentre, prospective study of $\mathrm{SCAP}$ suggest that $\mathrm{COPD}$ patients require mechanical ventilation 
TABLE 2 Initial antibiotic treatment and mortality rate in chronic obstructive pulmonary disease patients

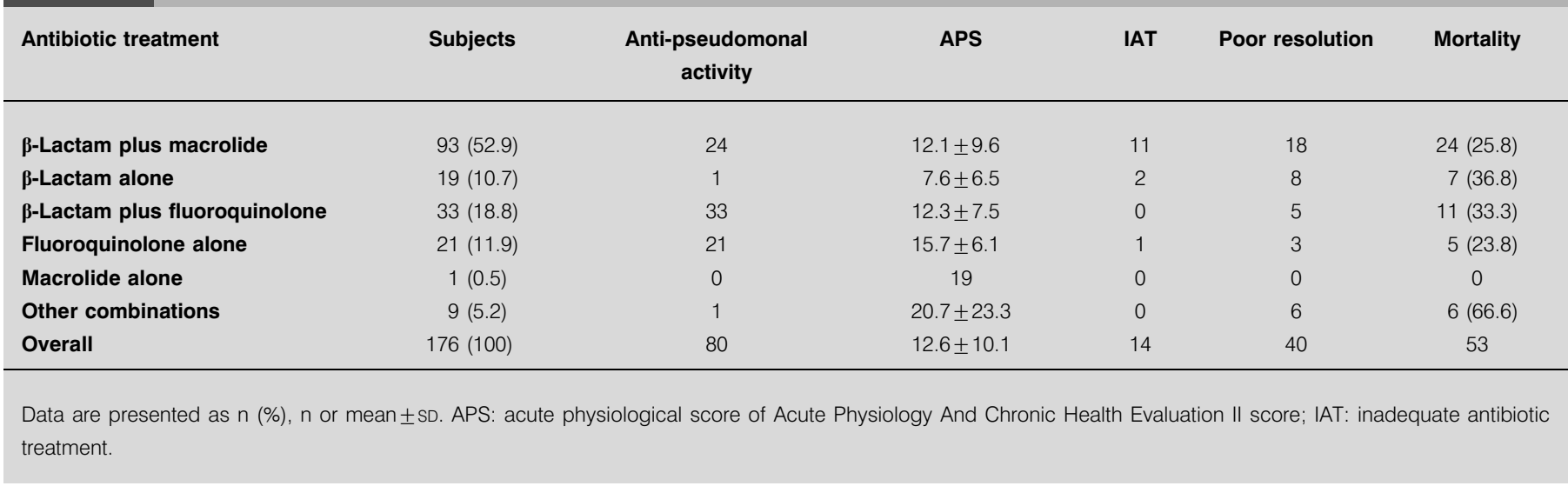

TABLE 3 Characteristics of 162 chronic obstructive pulmonary disease (COPD) patients with adequate empirical therapy who were ventilated $>24 \mathrm{~h}$

\begin{tabular}{lcccc} 
Variable & $\begin{array}{c}\text { COPD patients who died } \\
\text { during ICU stay }\end{array}$ & $\begin{array}{c}\text { COPD patients who survived } \\
\text { in ICU }\end{array}$ & p-value & OR (95\% CI) \\
\hline Subjects & 47 & 115 & \\
Age yrs & $69.0 \pm 7.7$ & $66.2 \pm 11.0$ & 0.11 \\
APS & $16.2 \pm 12.2$ & $10.8 \pm 8.7$ & $<0.01$ & 0.57 \\
Males & $39(82.9)$ & $101(87.8)$ & & 0.94 \\
Comorbidities & & & 0.67 & $1.09(0.54-2.21)$ \\
$\quad$ Smoker & $30(63.8)$ & $71(61.7)$ & 0.55 & $1.23(0.62-2.43)$ \\
Chronic heart disease & $22(46.8)$ & $48(41.7)$ & 0.01 & $1.34(0.64-2.77)$ \\
$\quad$ Alcoholism & $16(34.0)$ & $32(27.8)$ & $<0.01$ & $4.51(1.39-14.60)$ \\
$\quad$ Stroke & $8(17.0)$ & $5(4.3)$ & 0.70 & $15.80(5.77-43.00)$ \\
Shock & $42(89.4)$ & $40(34.7)$ & 0.04 & $0.81(0.08-8.01)$ \\
Empyema & $1(2.1)$ & $3(2.6)$ & $<0.01$ & $2.69(1.12-6.44)$ \\
Bacteraemia & $12(25.5)$ & $13(11.3)$ & $<0.01$ & $6.63(3.15-14.00)$ \\
Bilateral pneumonia & $31(66.0)$ & $26(22.6)$ & $\mathrm{NA}$ \\
Mechanical ventilation & $47(100.0)$ & $73(63.4)$ & & \\
\hline
\end{tabular}

Data are presented as $n$, mean \pm SD or $n(\%)$, unless otherwise stated. ICU: intensive care unit; OR: odds ratio; Cl: confidence interval; APS: acute physiological score of Acute Physiology And Chronic Health Evaluation II score; NA: not applicable.

more frequently, have a higher incidence of $P$. aeruginosa and higher ICU mortality rate than non-COPD patients. Patients with successful noninvasive mechanical ventilation had shorter ICU stays, but were significantly younger, with lower severity according to the APS at ICU admission, less radiographical involvement and fewer comorbidities (such as chronic heart failure). As reported elsewhere [12, 18], a delay in adequate empirical therapy due to unexpected in vitro resistance of the responsible pathogen was associated with excess mortality, whereas with an adequate empirical regimen only bilateral pneumonia and lower APACHE II score measured on the first day of ICU admission were associated with ICU mortality.

Previous studies in COPD patients have suggested that Chlamydia pneumoniae is a frequent cause of SCAP [11, 19]. In the present study, other atypical agents, such as Mycoplasma pneumoniae, or common organisms of the normal respiratory tract in COPD [20, 21], e.g. Moraxella catarrhalis, were infrequently identified. This is probably due to the difference of severity in episodes requiring ICU admission. S. pneumoniae remained the most frequent pathogen and the leading cause in fatal episodes, emphasising COPD patients should be administered anti-pneumococcal vaccinations. Nontypable $H$. influenzae was the third most prevalent pathogen, but an uncommon cause of death. Viruses are important agents of COPD exacerbation but none were identified, although systematic samples from nasal airways were not investigated.

$P$. aeruginosa was the second most common organism in patients with COPD and this is another aspect of interest. Moreover, incidence was significantly higher than in non-COPD patients and it appears to be a near exclusive problem in COPD patients. However, only $7.4 \%$ of COPD patients had $P$. aeruginosa and the current authors were unable to anticipate potential variables identifying COPD patients at high risk of $P$. aeruginosa. 


\begin{tabular}{|c|c|c|c|c|c|}
\hline TABLE 4 & \multicolumn{5}{|c|}{$\begin{array}{l}\text { Variables independently associated with } \\
\text { intensive care unit mortality in chronic obstructive } \\
\text { pulmonary disease (COPD) and non-COPD } \\
\text { patients using Cox proportional regression } \\
\text { analysis }\end{array}$} \\
\hline \multirow{2}{*}{\multicolumn{2}{|c|}{ Variable }} & \multicolumn{2}{|c|}{ COPD patients } & \multicolumn{2}{|c|}{ Non-COPD patients } \\
\hline & & OR (95\% Cl) & $p$-value & OR (95 \%Cl) & p-value \\
\hline \multicolumn{2}{|l|}{ APS per point } & & & $1.06(1.03-1.09)$ & $<0.001$ \\
\hline \multirow{2}{*}{\multicolumn{2}{|c|}{ Age per yr }} & & & $1.03(1.01-1.06)$ & 0.001 \\
\hline & & $2.32(1.18-4.53)$ & 0.01 & $2.23(1.06-4.67)$ & 0.03 \\
\hline \multicolumn{2}{|l|}{$\begin{array}{l}\text { Shock } \\
\text { Shock }\end{array}$} & $3.53(1.31-9.71)$ & 0.01 & $5.87(2.30-14.99)$ & $<0.001$ \\
\hline
\end{tabular}

OR: odds ratio; Cl: confidence interval; APS: acute physiological score of Acute Physiology And Chronic Health Evaluation II score.

The pulmonary severity index (PSI) defined from the Pneumonia Outcomes Research Team study [22] includes five comorbid conditions (cardiovascular, malignancy, cerebrovascular, renal and liver diseases), but does not include COPD as one of them. This score was designed in a cohort of patients who included a minority of patients requiring ICU admission and was primarily designed to influence the decision to hospitalise the patient. In addition, in the PSI score, COPD was analysed together with asthma and interstitial lung disease, which could be a reason why it was not identified as a risk factor for poor outcome. Another multicentre study in Spain [11] reported low overall mortality for COPD patients with pneumonia. However, all COPD patients with pneumonia

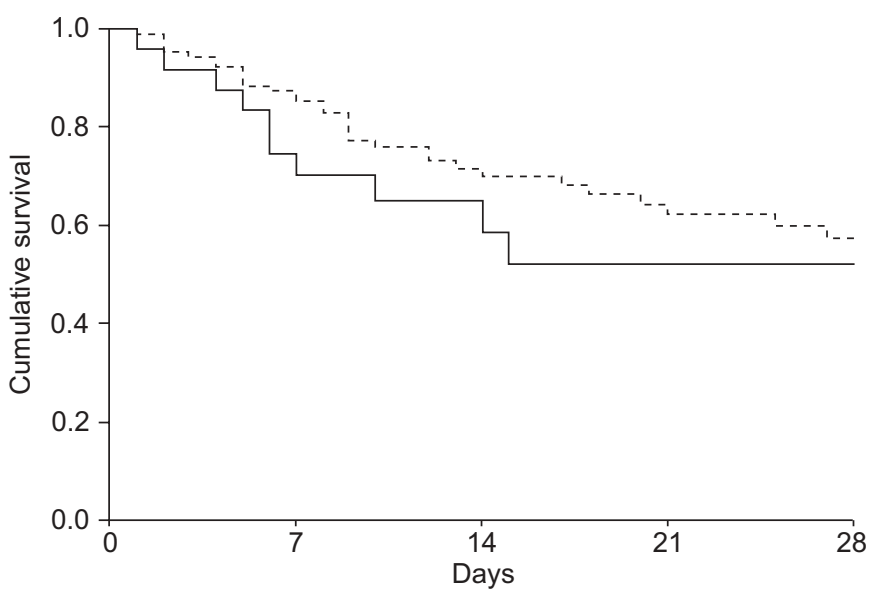

FIGURE 2. Kaplan-Meier survival curve for chronic obstructive pulmonary disease patients with initial intubation (- - -) versus delaying intubation (-) due to unsuccessful noninvasive ventilation.

were hospitalised and $<20 \%$ needed ventilatory support. In contrast, nearly $90 \%$ of the current cohort required mechanical ventilation. This group represents only a small proportion of hospitalised COPD patients, but it is the one with the highest mortality rates.

The present study confirms that it is crucial for physicians to initiate correct treatment if the risk of mortality is not to increase. The multivariate analysis failed to identify intervention-related factors that were able to improve survival in the subset receiving agents with adequate sensitivity. However, this study was not designed for this purpose. Moreover, factors other than the simple concept of in vitro

\begin{tabular}{|c|c|c|c|c|c|c|c|c|}
\hline \multirow[t]{2}{*}{ Variable } & \multicolumn{4}{|c|}{ COPD patients } & \multicolumn{4}{|c|}{ Non-COPD patients } \\
\hline & Initial intubation & Failure to NIV & Successful NIV & p-value ${ }^{\#}$ & Initial intubation & Failure to NIV & Successful NIV & p-value ${ }^{\#}$ \\
\hline Subjects & 105 & 24 & 26 & & 122 & 25 & 36 & \\
\hline ICU mortality rate & $41(39)$ & $12(50)$ & 0 & $<0.001$ & $41(33.6)$ & $9(36.0)$ & $3(8.3)$ & 0.01 \\
\hline LOS for survivors days & $17.7 \pm 14.5$ & $18.5 \pm 11.1$ & $8.2 \pm 7.1$ & $<0.001$ & $25.7 \pm 19.7$ & $31.1 \pm 26.2$ & $9.5 \pm 5.5$ & $<0.001$ \\
\hline \multicolumn{9}{|l|}{ Main comorbidities } \\
\hline Smoker & $67(63.8)$ & $16(66.7)$ & $14(53.8)$ & 0.52 & $51(41.8)$ & $8(32.0)$ & $12(33.3)$ & 1.00 \\
\hline Chronic heart disease & $47(44.8)$ & $14(58.3)$ & $7(26.9)$ & 0.05 & $30(24.6)$ & $7(28.0)$ & $8(22.2)$ & 0.76 \\
\hline Empyema & $2(1.9)$ & 0 & $1(3.8)$ & 1.0 & $9(7.4)$ & $1(4.0)$ & $4(11.1)$ & 0.65 \\
\hline Presence of $P$. aeruginosa & $9(8.6)$ & $3(12.5)$ & 0 & 0.10 & $1(0.8)$ & 0 & 0 & \\
\hline
\end{tabular}

Data are presented as $n$, mean \pm SD or $n(\%)$, unless otherwise stated. NIV: noninvasive ventilation; APS: acute physiologic score of Acute Physiology And Chronic Health Evaluation II score; ICU: intensive care unit; LOS: length of stay; $P$. aeruginosa: Pseudomonas aeruginosa. ${ }^{*}$ : p-value for comparison between patients with failure to NIV and successful NIV. 


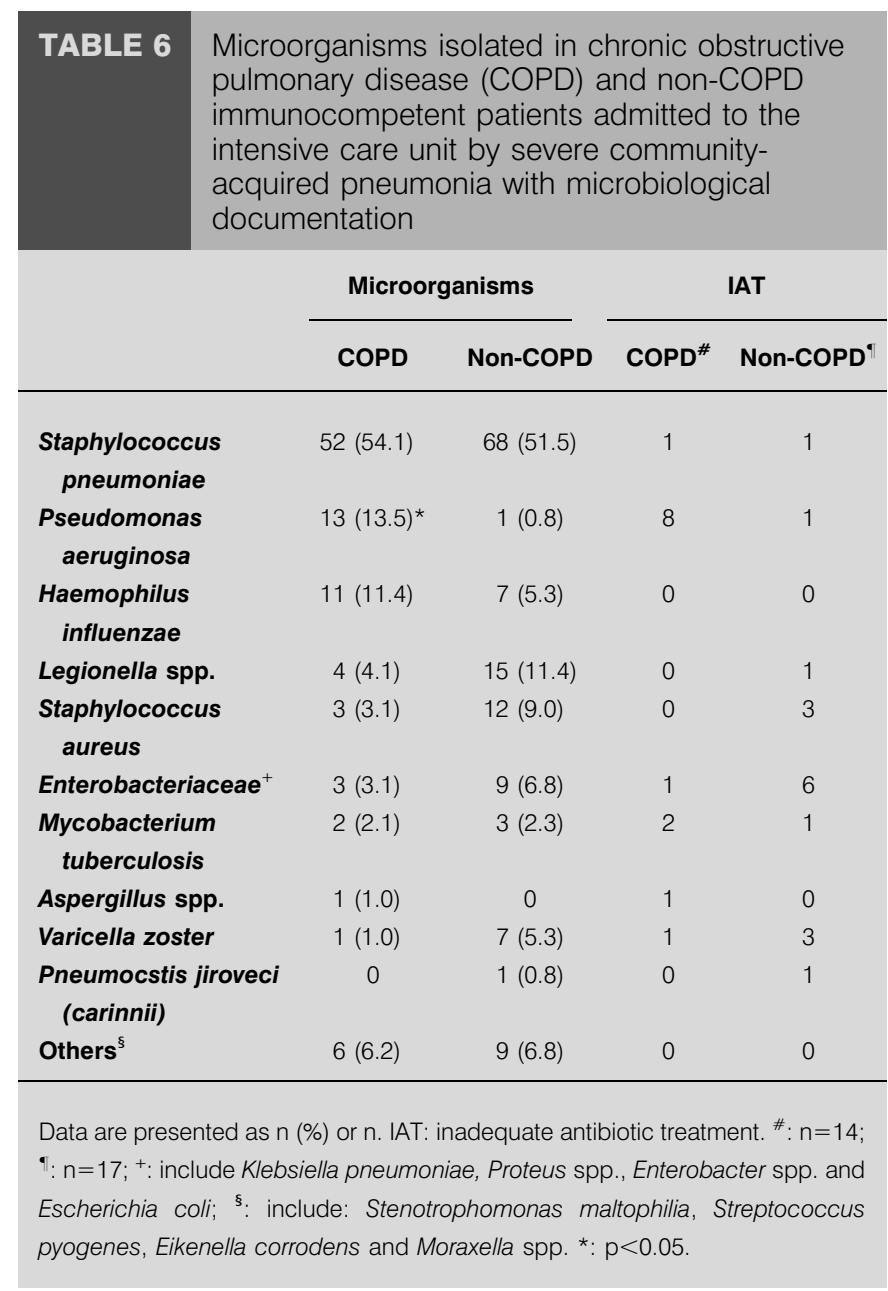

activity alone play key roles in fighting infection. WATERER et al. [23] suggested that monotherapy may be suboptimal for patients with bacteraemic S. pneumoniae pneumonia, whatever treatment is given. Further studies [24-27] agree with these findings, but the controversy should be resolved by conducting a randomised clinical trial.

There were some limitations to the current study. First, it was not possible to record data regarding lung function tests or COPD disease severity. Although they can be used to classify severity and assess clinical response, these tests are usually not suitable in ICU patients $[7,28]$. Further prospective cohort studies should determine the impact of COPD severity on survival and microbiology. Secondly, as in other studies exploring combination therapy, these results were not from a randomised controlled study. However, severity of illness measured by APS was identical. The present study cohort comprised patients with severe episodes of pneumonia requiring intensive care. Therefore, the current findings should not be extrapolated to COPD patients with pneumonia requiring hospitalisation in medical wards or with acute exacerbation. Immunocompromised patients were excluded and the current findings cannot be extrapolated to COPD patients with long-term oral steroid treatment, in whom the pathogen responsible may be an opportunistic microorganism. Patients included in the current study did not receive steroids in routine clinical practice, even those associated with septic shock. The effect of steroids in pneumonia should be further evaluated as part of randomised clinical trials. Finally, followup did not extend beyond the ICU stay and the continued drop in survival after ICU discharge is well documented [29]. However, the optimal time-frame for the ascertainment of mortality should be that during which the intervention can plausibly be expected to affect survival and should encompass the potential impact of the intervention. Indeed, short-term survival can be considered a surrogate measure. In addition, long-term survival is confounded by deaths from other causes [30].

In conclusion, the present study demonstrates significantly higher mortality rates among patients with chronic obstructive pulmonary disease who require intensive care unit admission for acute respiratory failure due to severe community-acquired pneumonia. This selected population of patients has a higher need for mechanical ventilation and a higher incidence of Pseudomonas aeruginosa than non-chronic obstructive pulmonary disease patients.

\section{ACKNOWLEDGEMENTS}

The following is a list of the CAPUCI study investigators. J. Solé: Dr Negrin Hospital, Gran Canaria; J. Blanquer: Clinic Hospital, Valencia; J. Jiménez: Virgen del Rocio, Sevilla; V. de la Torre, Virgen de la Victoria Hospital, Malaga; J.M. Sirvent: Josep Trueta Hospital, Girona; M. Bodí: Joan XXIII Hospital, Tarragona; J. Almirall: Mataró Hospital, Mataró, Barcelona; A. Doblas: Juan Ramon Jimenez Hospital, Huelva; J.R. Badía: Clinic Hospital Barcelona, Barcelona; F. García: General Hospital, Albacete; A. Mendia: Nuestra Señora de Aranzazu Hospital, San Sebastian; R. Jordá: Son Dureta Hospital, Palma de Mallorca; F. Bobillo: Clinico Hospital, Valladolid; J. Vallés: Hospital Parc Tauli, Sabadell, Barcelona; M.J. Broch: Sagunto Hospital, Valencia; N. Carrasco: Princesa Hospital, Madrid; M.A. Herranz: Rio Hortega Hospital, Valladolid; F. Alvarez Lerma: Del Mar Hospital, Barcelona; E. Mesalles: Trias I Pujol Hopsital, Badalona, Barcelona; B. Alvarez: General Hospital, Alicante; J.C. Robles: Reina Sofia Hospital, Córdoba; E. Maraví: Virgen del camino hospital, Pampalona; F. Barcenilla: Arnau de Vilanova Hospital, Lleida; M.A. Blasco: Peset Aleixandre Hospital, Valencia; G. Masdeu: Verge de la Cinta Hospital, Tortosa, Tarragona; M.J. López Pueyo: General de Yagüe Hospital, Burgos; A. Margarit: Virgen Meritxell Hospital, Andorra; J. Fierro: Poniente Hospital, Almeria; F. Renedo: Leon Hospital, Leon; A. Lores: Bellvitge Hospital, Barcelona; R. Alonso: General de Asturias Hospital, Oviedo; M.J. Huertos: Puerto Real Hospital, Cadiz; M.J. López Cambra: General Hospital, Segovia.

\section{REFERENCES}

1 Boersma WG, Sportel JH, Löwenberg A, Koëter GH. High prevalence of obstructive airways disease in hospitalized patients with community-acquired pneumonia: comparison of four etiologies. Clin Pulm Med 2005; 12: 291-296.

2 Marrie TJ, Durant H, Yates L. Community-acquired pneumonia requiring hospitalization: 5-year prospective study. Rev Infect Dis 1989; 2: 586-599.

3 Jansen HM, Sachs AP, van Alphen L. Predisposing conditions to bacterial infections in chronic obstructive 
pulmonary disease. Am J Respir Crit Care Med 1995; 151: 2073-2080.

4 Fekety FR, Caldwell J, Gump D, et al. Bacteria, viruses and mycoplasmas in acute pneumonia in adults. Am Rev Respir Dis 1971; 104: 499-507.

5 Ewig S, Ruiz M, Mensa JM, et al. Severe communityacquired pneumonia. Assessment of severity criteria. Am J Respir Crit Care Med 1998; 158: 1102-1108.

6 Restrepo MI, Anzueto A. Antimicrobial treatment of community-acquired pneumonia. Clin Chest Med 2005; 26: 65-73.

7 Almirall J, Bolibar I, Balanzo X, Gonzalez CA. Risk factors for community-acquired pneumonia in adults: a populationbased case-control study. Eur Respir J 1999; 13: 349-355.

8 Arancibia F, Bauer TT, Ewig S, et al. Community-acquired pneumonia due to Gram-negative bacteria and Pseudomonas aeruginosa: incidence, risk and prognosis. Arch Intern Med 2002; 162: 1849-1858.

9 Lujan M, Gallego M, Fontanals D, Mariscal D, Rello J. Association of discordant antibiotic therapy and mortality in adults with bacteremic pneumococcal communityacquired pneumonia. Crit Care Med 2004; 32: 625-631.

10 Falguera M, Pifarre R, Martín A, Sheikh A, Moreno A. Etiology and outcome of community-acquired pneumonia in patients with diabetes mellitus. Chest 2005; 128: 3233-3239.

11 Torres A, Dorca J, Zalacain R, et al. Community-acquired pneumonia in chronic obstructive pulmonary disease: a Spanish multicenter study. Am J Respir Crit Care Med 1996; 154: 1456-1461.

12 Bodi M, Rodríguez A, Solé-Violán J, et al. Antibiotic prescription for community-acquired pneumonia in the intensive care unit. Impact of adherence to IDSA guidelines on survival. Clin Infect Dis 2005; 41: 1709-1716.

13 Standards for the diagnosis and care of patients with chronic obstructive pulmonary disease (COPD) and asthma. American Thoracic Society. Am Rev Respir Dis 1987; 136: 225-244.

14 Rello J, Bodí M, Mariscal D, et al. Microbiological testing and outcome of patients with severe community-acquired pneumonia. Chest 2003; 123: 174-180.

15 Hilbert G, Gruson D, Vargas F, et al. Noninvasive ventilation in immunosuppressed patients with pulmonary infiltrates, fever and acute respiratory failure. $N$ Engl J Med 2001; 344: 481-487.

16 American Thoracic Society. Guidelines for the management of adults with community-acquired pneumonia. Am J Respir Crit Care Med 2001; 163: 1730-1754.

17 Mandell LA, Bartlett JG, Dowell SF, File TM, Musher DM, Whitney C. Update of practice guidelines for the management of community-acquired pneumonia in immunocompetent adults. Clin Infect Dis 2003; 37: 1405-1433.

18 Lujan M, Gallego M, Fontanals D, Mariscal D, Rello J. Prospective observational study of bacteremic pneumococcal pneumonia: Effect of discordant therapy on mortality. Crit Care Med 2004; 32: 625-631.

19 Sopena N, Pedro-Botet ML, Sabria M, et al. Comparative study of community-acquired pneumonia caused by Streptococcus pneumoniae, Legionella pnuemophila or Chlamydia pnuemoniae. Scand J Infect Dis 2004; 36: 330-334.

20 Monsó E, Ruiz J, Rosell A, et al. Bacterial infection in chronic obstructive pulmonary disease. Am J Respir Crit Care Med 1995; 152: 1316-1320.

21 Feldman C, Smith C, Kaka P, de Jong P, Prommitz DA. The clinical significance of Haemophilus influenzae and $H$. parainfluenzae isolated from the sputum of adult patients at an urban general hospital. S Afr Med J 1992; 81: 495-498.

22 Fine MJ, Auble TE, Yealy DM, et al. A predictive rule to identify low-risk patients with community-acquired pneumonia. N Engl J Med 1997; 336: 243-250.

23 Waterer GW, Somes GW, Wunderink RG. Monotherapy may be suboptimal for severe bacteremic pneumococcal pneumonia. Arch Intern Med 2001; 161: 1837-1842.

24 Martinez JA, Horcajada JP, Almela M, et al. Addition of macrolide to a $\beta$-lactam-based empirical antibiotic regimen is associated with lower in-hospital mortality for patients with bacteremic pneumococcal pneumonia. Clin Infect Dis 2003; 36: 289-295.

25 Weiss K, Low DE, Cortes L, et al. Clinical characteristics at initial presentation and impact of dual therapy on the outcome of bacteremic Streptococcus pneumoniae pneumonia in adults. Can Respir J 2004; 11: 589-593.

26 Baddour LM, Yu VL, Klugman KP, et al. Combination antibiotic therapy lowers mortality among severely ill patients with pneumococcal bacteremia. Am J Respir Crit Care Med 2004; 170: 440-444.

27 Waterer GW, Rello J. Choosing the right combination therapy in severe community-acquired pneumonia. Crit Care 2006; 10: 115.

28 Nevins ML, Epstein SK. Predictors of outcomes for patients with COPD requiring invasive mechanical ventilation. Chest 2001; 119: 1840-1849.

29 Waterer G, Kessler LA, Wunderink RG. Medium-term survival after hospitalization with community-acquired pneumonia. Am J Respir Crit Care Med 2004; 169: 910-914.

30 Marshall JC, Vincent JL, Guyatt G, et al. Outcome measures for clinical research in sepsis: a report of the 2nd Cambridge Colloquium of the International Sepsis Forum. Crit Care Med 2005; 33: 1708-1716. 